\title{
Discordancy in BRAF mutations among primary and metastatic melanoma lesions: clinical implications for targeted therapy
}

Joshua R Bradish ${ }^{1,4}$, Justin D Richey ${ }^{1,4}$, Kristin M Post ${ }^{1}$, Kari Meehan ${ }^{1}$, Joyashree D Sen ${ }^{1}$, Amanda J Malek ${ }^{1}$, Terrence M Katona ${ }^{1,2}$, Simon Warren ${ }^{1}$, Theodore F Logan ${ }^{3}$, Leslie A Fecher ${ }^{3}$ and Liang Cheng ${ }^{1}$

${ }^{1}$ Department of Pathology, Indiana University School of Medicine, Indianapolis, IN, USA; ${ }^{2}$ Department of Pathology, Richard L Roudebush Veteran's Affairs Medical Center, Indianapolis, IN, USA and ${ }^{3}$ Department of Medicine, Indiana University School of Medicine, Indianapolis, IN, USA

\begin{abstract}
Systemic targeted molecular therapy, in the form of a selective BRAF inhibitor with or without a MEK inhibitor, is a standard treatment for patients with BRAF V600 mutation-positive melanoma with unresectable stage III and IV disease. Patients with BRAF mutation-negative primary tumors may manifest BRAF mutation-positive metastatic disease. It is unclear whether all metastatic lesions carry the same BRAF mutation status found in the primary tumor and if discordancy exists, in what frequency it occurs. Primary and matched metastatic lesions in 25 melanoma patients were tested for the BRAF V600E/Ec, V600K, V600D, and V600R mutations using a BRAF RGQ PCR kit (Qiagen). Four patients (16\%) had discrepancies between their primary and metastatic melanoma BRAF status. Of these patients, $2(8 \%)$ had BRAF mutation-positive primary melanomas with BRAF mutation-negative metastatic lesions and $2(8 \%)$ patient had BRAF mutation-negative melanoma with a BRAF mutation-positive metastatic lesion. In summary, discordancy of BRAF mutation status is not an infrequent finding between primary and metastatic melanoma. It may be prudent in previously negative patients to determine BRAF mutation status of new metastatic tumors for proper allocation of BRAF inhibitor therapy. Discordant BRAF status may have a role in the varying patterns of response and inevitable resistance seen with BRAF inhibitor therapies.
\end{abstract}

Modern Pathology (2015) 28, 480-486; doi:10.1038/modpathol.2014.136; published online 7 November 2014

Approximately $2 \%$ of all individuals born in the United States today will develop melanoma. Of these, $16 \%$ will present with regional or distant metastasis. Unfortunately, the 5-year survival rate for patients at these stages is 62.4 and $16 \%$, respectively. ${ }^{1}$ The incidence of malignant melanoma has steadily risen over the past few decades. Early metastasis along with a propensity for afflicting a younger population causes a substantial number of years of potential life lost. Melanoma has also notoriously been refractory to chemotherapy regimens, with dacarbazine being the only FDA-approved

Correspondence: Professor L Cheng, MD, Department of Pathology, Indiana University School of Medicine, 350 West 11th Street, Rm 4010, Indianapolis, IN 46202, USA.

E-mail: liang_cheng@yahoo.com

${ }^{4}$ These authors contributed equally to this work.

Received 14 February 2014; revised 13 June 2014; accepted 8 August 2014; published online 7 November 2014 chemotherapy for the treatment of malignant melanoma. The arrival of BRAF inhibitors, namely vemurafenib and dabrafenib, transformed the way in which patients with malignant melanoma were treated. BRAF inhibitors demonstrate increased affinity for mutated BRAF enzymes over wild type and in this manner they selectively inhibit mutant melanocytes. BRAF mutations are found in $\sim 50 \%$ of malignant melanomas, especially those arising on non-chronically sun-damaged skin. ${ }^{2-4}$ When treated with BRAF inhibitors, patients have a significant improvement in progression-free survival as well as overall survival. ${ }^{5-7}$ In phase III trials comparing vemurafenib with dacarbazine therapy, vemurafenibtreated patients had a $48 \%$ response rate compared with only a $5 \%$ response rate in the dacarbazinetreated patients. In addition, there was a $63 \%$ relative reduction in the risk of death. ${ }^{6}$ Therefore, correctly identifying those patients who may derive some benefit through BRAF inhibitor therapy is paramount. 
Many methods only detect V600E mutations of the BRAF gene. Newer detection kits identify several less common V600 variant mutations, rather than only identifying V600E. Moreover, there is evidence that many less frequent BRAF mutations are sensitive to BRAF inhibitor or MEK inhibitor therapy., ${ }^{5,7-11}$ Currently those patients with unresectable stage III and IV melanoma are suggested to undergo BRAF mutation analysis and potential molecularly targeted therapy. The analysis, however, typically only takes place on one tissue block from one lesion with the assumption that all lesions from that patient will display similar BRAF status findings. The overall extent and impact of alternate BRAF mutations and discordancy is largely unknown. ${ }^{12-14}$ If a significant proportion of patients demonstrate intertumoral discordancy, they may benefit from BRAF inhibitor therapy, which would have not been offered to them initially. In addition, the detection of other less common BRAF mutations would also increase the number of patients offered a BRAF and/or MEK inhibitor. To this end, we studied 25 patients with both primary and metastatic diseases to determine the intertumoral discordance and detection of less frequent BRAF V600 mutations.

\section{Materials and methods}

Tissue samples from 177 patients with unresectable and/or metastatic melanoma were sent for molecular $B R A F$ testing between May 2011 and May 2013. Of these, 25 patients with tumor tissue from both primary and metastatic sites were available for further testing. A patient chart review revealed no patients presented with multiple primary melanomas. Patients were $54 \%$ female and $46 \%$ male with an age ranging from 23 to 81 years. Tissue tested included primary skin, skin metastasis, lymph node metastasis, and one metastatic lesion to the larynx (Table 1). Genomic DNA was extracted using the Qiagen FFPE DNA extraction kit from six sections of formalin-fixed paraffin-embedded material $(5 \mathrm{mM}$ thickness each) from a total of 58 tumor samples in formalin-fixed paraffin-embedded tissue. To increase tumor cell population, hematoxylin and eosin slides were microscopically evaluated and marked by a pathologist. Marked areas containing tumor were subsequently microdissected. The area of the slides was at least $0.5 \mathrm{~cm}^{2}$ in general. The limit of detection was $2 \%$ for the assays. The DNA concentration was determined using the NanoDrop

Table 1 Comparative data for BRAF status evaluation in primary and metastatic sites ${ }^{\mathrm{a}}$

\begin{tabular}{|c|c|c|c|c|}
\hline Age (sex) & Primary (location) & Metastasis (location, multiple sites) & $\begin{array}{l}\text { Interim } B R A F \\
\text { inhibitor } \\
\text { treatment }\end{array}$ & Discordant \\
\hline $35(\mathrm{~F})$ & V600E/Ec (upper back) & V600E/Ec (brain) & No & No \\
\hline $26(\mathrm{M})$ & V600E/Ec (central back) & V600E/Ec (shoulder) & No & No \\
\hline $23(\mathrm{~F})$ & V600E/Ec (back) & V600E/Ec (brain) & No & No \\
\hline $38(\mathrm{M})$ & V600E/Ec (mid back) & V600E/Ec (left chest) & No & No \\
\hline $29(\mathrm{~F})$ & V600E/Ec (left leg) & V600E/Ec (left thigh) & No & No \\
\hline N/A & V600E/Ec (N/A) & V600E/Ec (regional lymph node) & N/A & No \\
\hline $57(\mathrm{M})$ & V600E/Ec (left thigh) & V600E/Ec (left pelvic bone) & No & No \\
\hline $44(\mathrm{M})$ & V600E/Ec (right upper back) & V600E/Ec (right posterior neck) & No & No \\
\hline $69(\mathrm{~F})$ & V600E/Ec (central back) & V600E/Ec (right back) & No & No \\
\hline $54(\mathrm{M})$ & V600E/Ec (abdomen) & V600E/Ec (left lower chest wall) & No & No \\
\hline $19(\mathrm{~F})$ & V600E/Ec (left shoulder) & V600E/Ec (left axilla) & No & No \\
\hline $60(\mathrm{~F})$ & V600E/Ec (right foot) & V600E/Ec (right thigh) & No & No \\
\hline $81(\mathrm{M})$ & WT (right ear) & WT (left lower lung) & No & No \\
\hline $55(\mathrm{M})$ & WT (occipital scalp) & WT (adrenal gland) & No & No \\
\hline $49(\mathrm{~F})$ & V600K (larynx, supraglottic) & V600K (lung, lingula) & No & No \\
\hline $69(\mathrm{M})$ & WT (right shoulder) & WT (parotid/neck) & No & No \\
\hline $55(\mathrm{~F})$ & V600K (right breast) & V600K (left inguinal node) & No & No \\
\hline $68(\mathrm{~F})$ & WT (right vulva) & WT (brain), WT (right inguinal node) & No & No \\
\hline $65(\mathrm{M})$ & V600E/Ec (right back) & V600E/Ec (scapula), V600E/Ec (right Axilla) & No & No \\
\hline $48(\mathrm{~F})$ & WT (left lower eyelid) & $\begin{array}{l}\text { WT (lung, right middle lobe), WT (right } \\
\text { pectoralis) }\end{array}$ & No & No \\
\hline $69(\mathrm{~F})$ & WT (left thumb) & $\begin{array}{l}\text { WT (axillary lymph node), WT axillary } \\
\text { lymph node) }\end{array}$ & No & No \\
\hline $47(\mathrm{~F})$ & V600E/Ec (right thigh) & $\begin{array}{l}\text { WT (right inguinal node), V600E (right } \\
\text { inguinal } \\
\text { node), V600E (right inguinal node) }\end{array}$ & No & Yes \\
\hline $29(\mathrm{~F})$ & WT (right upper arm) & V600E/Ec (left back), WT (N/A) & No & Yes \\
\hline $31(\mathrm{M})$ & WT (upper back) & V600E/Ec (axillary lymph node) & No & Yes \\
\hline $44(\mathrm{M})$ & V600E/Ec (right back) & WT (brain) & No & Yes \\
\hline Females $(n=13)$ & V600E/Ec $(n=15) ;$ & V600E/Ec $(n=15)$; & & $16 \%$ \\
\hline Males $(n=12)$ & V600K $(n=2) ;$ WT $(n=8)$ & V600K $(n=2) ;$ WT $(n=8)$ & & Discordant \\
\hline
\end{tabular}

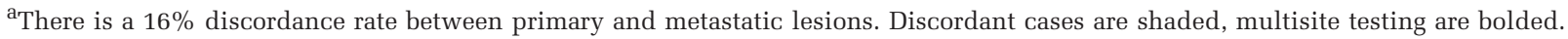


Spectrophotometer then placed on the BRAF assay. The assay detects five somatic mutations in the $B R A F$ gene using real-time PCR on the Rotor-Gene $\mathrm{Q}$ 5plex HRM instrument. The assay uses ARMS (Amplification Refractory Mutation System) and Scorpions technologies; enabling detection of the following mutations at codon 600 of the BRAF oncogene against a background of wild-type genomic DNA: V600E/V600E complex (V600Ec), V600D, V600K, and V600R. The control assay, labeled with FAM, is used to assess the total amplifiable BRAF DNA in a sample. The control assay amplifies a region of exon 3 of the $B R A F$ gene. The primers and
Scorpion probe have been designed to amplify independently of any known BRAF polymorphisms. All procedures were performed according to the manufacturer's protocol (Qiagen Manchester, Manchester, UK) (Figure 1).

Furthermore, a patient chart review revealed that no patients were treated with BRAF inhibitor therapy between primary and metastatic lesion resection. There was one patient for whom clinical information was not available; however, this patient did not demonstrate discordancy (Table 1). This research was approved by the Institutional Review Board.
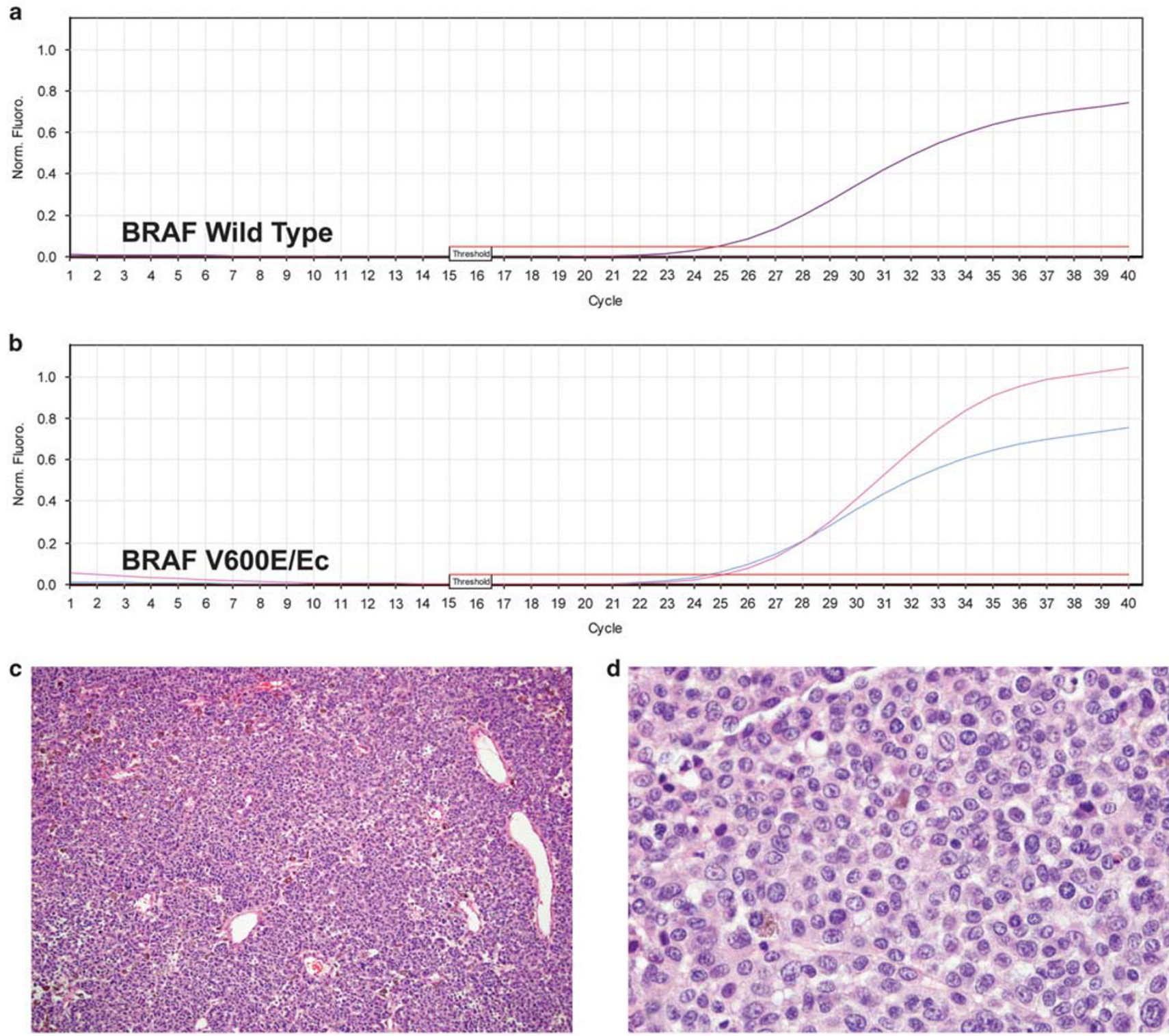

Figure 1 Qiagen results and histologic findings. (a) Raw fluorescence of a mutation-negative sample. Sample control (purple) in the FAM channel true amplification within the acceptable specified range. No amplification present in the FAM channel for any of the mutations. (b) Raw fluorescence of a V600E/Ec sample. Sample control (purple) in the FAM channel true amplification within the acceptable specified range. Amplification for the V600E/Ec mutation acceptable (blue) and the calculated delta CT values lower than the cutoff value for V600E/Ec. (c) Low-power view of V600E metastatic melanoma. (d) High-power view of a V600E metastatic melanoma. 


\section{Results}

We analyzed the BRAF mutations in both primary and metastatic sites from 25 melanoma patients. Patients were $54 \%$ female and $46 \%$ male with an age ranging from 23 to 81 years. Tissue tested included primary skin, skin metastasis and lymph node metastasis, and one metastatic lesion to the larynx.

BRAF status and anatomic tumor location, along with patient demographics, were compiled and are included in Table 1. BRAF status was determined in all 58 specimens from 25 patients in which 37 (64\%) were positive for a BRAF mutation (Table 1). Four of the $25(16 \%)$ patients had BRAF mutation discordancy between the primary and metastatic lesions. Of the discordant cases, two (8\%) had BRAF V600 mutation-positive primary melanomas with wildtype metastatic lesions and two (8\%) patients had a BRAF V600 wild-type primary melanoma with a V600 mutation-positive metastatic lesion (Table 2). Tissue from multiple metastatic sites was available for testing in six patients. A much higher discordancy rate of two out of the six $(33 \%)$ was found when multiple sites were tested (Table 3).

\section{Discussion}

Early studies on BRAF heterogeneity proposed that testing of either the primary or the metastatic lesion was sufficient as little discordance existed between different tumor sites. ${ }^{15,16}$ More recently, BRAF status discrepancies between primary and metastatic sites range from 18 to $26 \% .{ }^{13}$ We also identify the frequent presence of BRAF discordancy between

Table 2 Variable occurrence in discordancy of BRAF mutations ${ }^{\mathrm{a}}$

\begin{tabular}{llc}
\hline Primary lesion & Metastatic lesion & Number of cases \\
\hline Wild type & Mutated & 2 \\
Mutated & Wild type & 2 \\
Wild type & Wild type & 6 \\
Mutated & Mutated & 15 \\
Total & & 25 \\
\hline
\end{tabular}

${ }^{\mathrm{a} B o t h}$ wild-type and mutated discordances were identified.

Table 3 Rate of discordancy in multisite testing ${ }^{\mathrm{a}}$

\begin{tabular}{lccc}
\hline Scenario & $\begin{array}{c}\text { Number } \\
\text { tested }\end{array}$ & $\begin{array}{c}\text { Number } \\
\text { discordant }\end{array}$ & $\begin{array}{c}\% \\
\text { Discordant }\end{array}$ \\
\hline Single metastasis & 19 & 2 & $11 \%$ \\
Multiple metastasis & 6 & 2 & $33 \%$ \\
\hline
\end{tabular}

${ }^{\mathrm{a}}$ Increasing the number of lesions tested per patient increased the discordancy findings. primary and metastatic melanoma lesions of $16 \%$ (Table 1). In addition, we find variability in discordance, that is, a wild-type primary tumor with a mutant metastasis and vice versa (Table 2). Moreover, we show that testing multiple sites in a single patient increases the likelihood of finding BRAF discrepancies (Table 3). Improved patient survival rates with BRAF inhibitors suggest that every opportunity should be taken to detect these mutations when they are present. One major limitation of the current study includes a relatively small number of patients who qualified (multiple tumors, tissue available for testing, etc) even after a broad search was performed. Also, there is a real possibility that other mutations outside the ones identified by the Qiagen test could have been present and not identified as only the most common mutations would be identified $(<1 \%)$.

Not surprisingly, patients with BRAF V600 mutation-negative melanoma do not derive any benefit from BRAF inhibitor therapy. ${ }^{17,18}$ In fact, paradoxically, BRAF V600 mutation-negative tumor cells treated with selective BRAF inhibitors exhibited an increase rate of proliferation, reduced cell adherence, and increased mobility of cells. ${ }^{19}$ The intertumoral and intratumoral heterogeneity may partially account for the range in initial patient response as well as timing and manifestations of resistance. For maximum patient benefit as well as proper allocation of resources, patients need to be properly classified; including those with less frequent BRAF mutations. The second most common mutation is the BRAF V600K mutation, representing $14 \%$ to $28 \%$ of $B R A F$ mutations in melanoma. ${ }^{20}$ We discovered two V600K mutations, which were detected only after using the Qiagen kit, as opposed to the older detection methods (Table 1). This is especially important, as there is emerging literature that many less common BRAF mutations are also responsive to inhibitor therapy including V600K, V600R, K601E, and L597S. ${ }^{5,8-11,21,22}$ In our opinion, through optimizing the identification of different types of BRAF mutations, those patients with less frequent BRAF mutations may have access to this life-extending class of drugs, who would have otherwise been excluded from selective BRAF or MEK inhibitor therapy. Additionally, development of therapeutic agents with varied spectrums of BRAF (and MAPK pathway) inhibition may lead to more therapeutic options for patients with nonV600E BRAF aberrations. ${ }^{23}$ To this end, more updated and advanced detection methods should be encouraged.

Discordancy is frequently present between primary and metastatic lesions of patients with melanoma. Multiple mechanisms may be responsible for these discrepancies (Figure 2). We demonstrate that testing multiple metastatic sites increased the percentage of discordant cases as well as BRAF mutations overall. The clinical and prognostic 

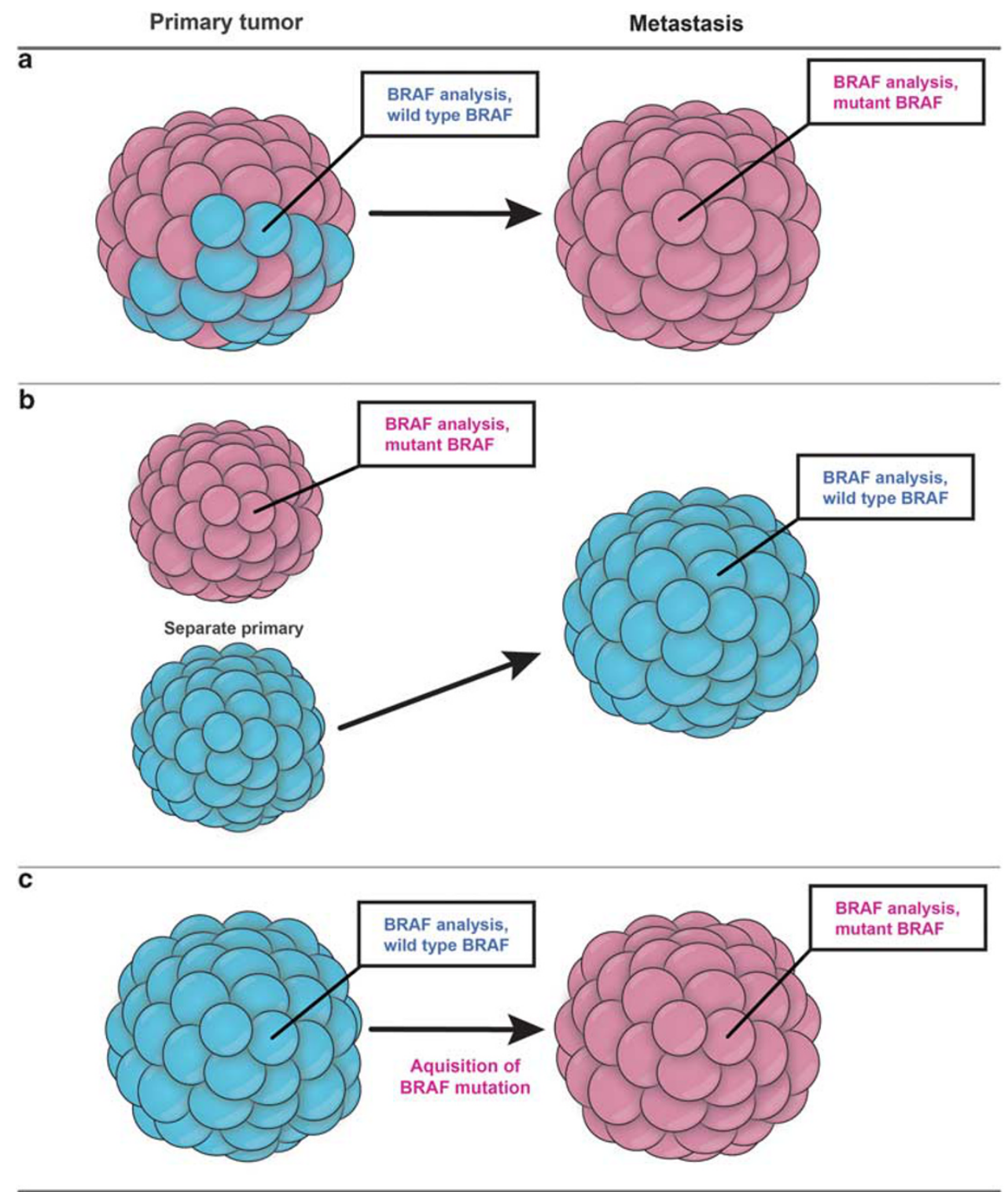

= wild type

$=$ mutant

Figure 2 Mechanisms of discordant results in BRAF mutation analysis. (a) Intratumoral heterogeneity may show a wild-type primary analysis, yet the metastatic lesions may arise from the mutated cells. The reverse findings may also be seen (ie, a mutated primary analysis and wild-type metastasis). (b) Two separate primary melanomas may yield discordant results if the first primary is tested while the second gives rise to metastasis. (c) Melanoma cells may acquire BRAF mutations over time, evident in metastatic lesions but not present in the primary. At the time of primary excision, already metastatic melanocytes may acquire BRAF mutations after analysis of the primary lesion.

significance of discordancy is currently unknown. Recent studies evaluating immunohistochemical stains for V600E-mutated BRAF reveals a sensitivity and specificity of 97 and $100 \%$, respectively. Immunohistochemistry was, however, less sensitive in detecting V600 mutations overall (91\%). ${ }^{12,24}$ If immunohistochemistry proves to be a valid method of BRAF detection, standardization will provide more interobserver uniformity. This may allow for easier assessment of intra- as well as intertumoral heterogeneity and discordancy, respectively. There are conflicting findings among the few papers specifically addressing BRAF heterogeneity in melanoma. One recent paper found no intertumoral heterogeneity between primary and metastatic lesions. ${ }^{25}$ Whereas these findings are at odds with our results, multiple other studies are in agreement with our reported findings. ${ }^{12,14,26,27}$ The main difference between these previous studies and our present findings is that we have identified discordancy from both mutated and wild-type primary lesions. 
Current treatments and strategies only focus on interpatient heterogeneity but there is a growing understanding that perhaps a focus on intratumor and intertumor variability within the same patient should be the new approach. ${ }^{28}$ Current strategies do not account for evolution of tumors over time, which may have a role in the discrepancy in BRAF in melanoma, and possibly in other genes in melanoma and other cancers. Previous studies from our group demonstrated that significant genetic divergence occurs frequently during the clonal evolution of melanoma and that multiple coexisting metastases appear to be derived from different, genetically unrelated tumor clones. ${ }^{27}$ Perhaps the evaluation and treatment of melanoma should broaden to accommodate the theory of tumoral heterogeneity and that tumors may evolve over time. In other tumors such as breast carcinoma, the continual monitoring of estrogen, progesterone, and HER2/neu receptors over time is standard of care. In lymphoproliferative disorders it is not uncommon to assess progression and therapeutic strategy based on molecular and immunohistochemical evolution over time. The evidence of discrepant BRAF mutation findings may account for the large variability in response among patients with BRAF inhibitor-treated melanoma. Future studies evaluating the optimal therapeutic strategy for patients with disparate BRAF mutational status are necessary.

\section{Disclosure/conflict of interest}

The authors declare no conflict of interest.

\section{References}

1 Howlader N, Noone AM, Krapcho M, et al. SEER cancer statistics review, 1975-2010, National Cancer Institute. Bethesda, MD, USA. http://seer.cancer.gov/ csr/1975_2010/. based on November 2012 SEER data submission, posted to the SEER website, April 2013.

2 Cheng L, Eble JN. Molecular Surgical Pathology, 1st edn. Springer: New York, NY, USA, 2013.

3 Cheng L, Zhang DY, Eble JN. Molecular Genetic Pathology, 2nd edn. Springer: New York, NY, USA, 2013.

4 Bradish JR, Cheng L. Molecular pathology of malignant melanoma: changing the clinical practice paradigm toward a personalized approach. Hum Pathol 2014;45:1315-1326.

5 Sosman JA, Kim KB, Schuchter L, et al. Survival in BRAF V600-mutant advanced melanoma treated with vemurafenib. N Engl J Med 2012;366:707-714.

6 Chapman PB, Hauschild A, Robert C, et al. Improved survival with vemurafenib in melanoma with BRAF V600E mutation. N Engl J Med 2011;364:2507-2516.

7 Bradish JR, Montironi O, Lopez-Beltran A, et al. Towards personalized therapy for patients with malignant melanoma: molecular insights into the biology of BRAF mutations. Future Oncology 2013;9:245-253.
8 Bahadoran P, Allegra M, Le Duff F, et al. Major clinical response to a BRAF inhibitor in a patient with a BRAF L597R-mutated melanoma. J Clin Oncol 2013;31: e324-e326.

9 Rubinstein JC, Sznol M, Pavlick AC, et al. Incidence of the V600K mutation among melanoma patients with BRAF mutations, and potential therapeutic response to the specific BRAF inhibitor PLX4032. J Transl Med 2010;8:67.

10 Falchook GS, Long GV, Kurzrock R, et al. Dabrafenib in patients with melanoma, untreated brain metastases, and other solid tumours: a phase 1 dose-escalation trial. Lancet 2012;379:1893-1901.

11 Dahlman KB, Xia J, Hutchinson K, et al. BRAF(L597) mutations in melanoma are associated with sensitivity to MEK inhibitors. Cancer Discov 2012;2: 791-797.

12 Busam KJ, Hedvat C, Pulitzer M, et al. Immunohistochemical analysis of BRAF(V600E) expression of primary and metastatic melanoma and comparison with mutation status and melanocyte differentiation antigens of metastatic lesions. Am J Surg Pathol 2013;37:413-420.

13 Heinzerling L, Baiter M, Kuhnapfel S, et al. Mutation landscape in melanoma patients clinical implications of heterogeneity of BRAF mutations. Br J Cancer 2013;109:2833-2841.

14 Yancovitz M, Litterman A, Yoon J, et al. Intra- and inter-tumor heterogeneity of BRAF(V600E))mutations in primary and metastatic melanoma. PLoS One 2012;7:e29336.

15 Omholt K, Platz A, Kanter L, et al. NRAS and BRAF mutations arise early during melanoma pathogenesis and are preserved throughout tumor progression. Clin Cancer Res 2003;9:6483-6488.

16 Colombino M, Capone M, Lissia A, et al. BRAF/NRAS mutation frequencies among primary tumors and metastases in patients with melanoma. J Clin Oncol 2012;30:2522-2529.

17 Tsai J, Lee JT, Wang W, et al. Discovery of a selective inhibitor of oncogenic B-Raf kinase with potent antimelanoma activity. Proc Natl Acad Sci USA 2008;105:3041-3046.

18 Joseph EW, Pratilas CA, Poulikakos PI, et al. The RAF inhibitor PLX4032 inhibits ERK signaling and tumor cell proliferation in a V600E BRAF-selective manner. Proc Natl Acad Sci USA 2010;107:14903-14908.

19 Halaban R, Zhang W, Bacchiocchi A, et al. PLX4032, a selective BRAF(V600E) kinase inhibitor, activates the ERK pathway and enhances cell migration and proliferation of BRAF melanoma cells. Pigment Cell Melanoma Res 2010;23:190-200.

20 Spittle C, Ward MR, Nathanson KL, et al. Application of a BRAF pyrosequencing assay for mutation detection and copy number analysis in malignant melanoma. J Mol Diagn 2007;9:464-471.

21 Kim KB, Kefford R, Pavlick AC, et al. Phase II study of the MEK1/MEK2 inhibitor Trametinib in patients with metastatic BRAF-mutant cutaneous melanoma previously treated with or without a BRAF inhibitor. J Clin Oncol 2013;31:482-489.

22 Ascierto PA, Minor D, Ribas A, et al. Phase II trial (BREAK-2) of the BRAF inhibitor dabrafenib (GSK2118436) in patients with metastatic melanoma. J Clin Oncol 2013;31:3205-3211.

23 Nakamura A, Arita T, Tsuchiya S, et al. Antitumor activity of the selective pan-RAF inhibitor TAK-632 in 
BRAF inhibitor-resistant melanoma. Cancer Res 2013; 73:7043-7055.

24 Boursault L, Haddad V, Vergier B, et al. Tumor homogeneity between primary and metastatic sites for BRAF status in metastatic melanoma determined by immunohistochemical and molecular testing. PLoS One 2013;8:e70826.

25 Menzies AM, Lum T, Wilmott JS, et al. Intrapatient homogeneity of BRAFV600E expression in melanoma. Am J Surg Pathol 2014;38:377-382.
26 Wilmott JS, Long GV, Howle JR, et al. Selective BRAF inhibitors induce marked T-cell infiltration into human metastatic melanoma. Clin Cancer Res 2012;18: 1386-1394.

27 Katona TM, Jones TD, Wang M, et al. Genetically heterogeneous and clonally unrelated metastases may arise in patients with cutaneous melanoma. Am J Surg Pathol 2007;31:1029-1037.

28 Bedard PL, Hansen AR, Ratain MJ, et al. Tumour heterogeneity in the clinic. Nature 2013;501:355-364. 